\title{
Management of female urinary incontinence
}

\section{Part 1 - Basics}

\author{
Ruwan J Fernando ${ }^{1}$
}

Sri Lanka Journal of Obstetrics and Gynaecology 2011; 33: 129-131

\section{Introduction}

This is the first of a series of review articles on recent developments in the management of female urinary incontinence. This review addresses the basics including the terminology and initial patient evaluation.

Urinary Incontinence (UI) is a common symptom that affects women of all ages with a wide range of severity and nature of symptoms. UI is not a lifethreatening disease, but the symptoms may seriously influence the physical, psychological, and social well being of the affected individuals. With increasing life expectancy and epidemic of obesity there has been a gradual rise in the prevalence of urinary incontinence worldwide. UI is defined as involuntary leakage of urine that is a social or hygienic problem ${ }^{1}$. Worldwide reported prevalence of urinary incontinence amongst adult women varies between $17-45 \%$. The wide range in the prevalence is partly due to variation of definitions, study population and survey methodology. Limited epidemiological studies show that the prevalence of UI among South East Asian females is comparable to worldwide figures ${ }^{2}$.

\section{Terminology and definitions}

In 2002 the International Consultation on Incontinence (ICI) published the terminology and definitions of lower urinary tract symptoms (LUTS) and is now accepted worldwide for clinical use and research purposes. The definitions are based on clinical features as well as urodynamic findings ${ }^{3}$.

Based on the micturition cycle, LUTS are mainly divided in to storage phase disorders and voiding phase disorders. Main storage phase symptoms are overactive bladder (OAB) symptoms, stress urinary incontinence (SUI), urge urinary incontinence (UUI) and mixed urinary incontinence (MUI). Other storage

\footnotetext{
${ }^{1}$ Consultant Obstetrician and Urogynaecology Subspecialist, Honorary Clinical Senior Lecturer, Imperial College Healthcare NHS Trust, St. Mary's Hospital, London W2 1NY UK.

Correspondence: Ruwan Fernando

E-mail: ruwanfernando@hotmail.com
}

symptoms include overflow incontinence and coital incontinence. Storage symptoms are primarily caused by overactivity of the detrusor muscle or under activity of the outlet (urethral sphincter).

Voiding phase symptoms includes poor flow, intermittent flow, hesitancy, straining and terminal dribbling. These symptoms are usually caused by the underactivity of the detrusor muscle and/or overactivity or obstruction to the outlet caused by pelvic organ prolapse, urethral stricture or following previous bladder neck surgery. Detrusor atonia or hypotonia is much more common in the female, and may arise idiopathically, or secondarily to over distension after childbirth or surgery, in peripheral neuropathy due to diabetes mellitus, and in other neurological conditions. Women with prolapse may require to digitate vaginally to initiate or complete voiding.

Overactive bladder (OAB): presence of urgency and frequency (either daytime or night-time), usually with frequency and nocturia in the absence of an underlying metabolic or pathological condition ${ }^{3}$. OAB is often divided into $\mathrm{OAB}$ without $\mathrm{UI}$ (OAB-dry) and those with $\mathrm{OAB}$ and $\mathrm{UI}$ (OAB-wet).

Stress urinary incontinence (SUI): complaint of involuntary leakage on effort or exertion, or on sneezing or coughing.

Urge urinary incontinence (UUI): complaint of involuntary leakage accompanied by or immediately preceded by urgency. Urge incontinence can present in different symptomatic forms; for example, as frequent small losses between micturition or as a sudden leak with complete bladder emptying. Information should be sought on triggering events such as cold, running water and 'latch key' incontinence ${ }^{3}$.

Mixed urinary incontinence (MUI): complaint of involuntary leakage associated with urgency and also with exertion, effort, sneezing or coughing ${ }^{3}$.

Other types of UI may be situational, for example the report of incontinence during sexual intercourse. Coital incontinence may occur during arousal, on 
penetration, throughout intercourse, or specifically at orgasm. Although urodynamic stress incontinence is the most common urodynamic finding in each of these situations, detrusor overactivity is found more often when leakage is restricted to orgasm ${ }^{3}$.

Urinary urgency: complaint of a sudden compelling desire to pass urine which is difficult to defer ${ }^{3}$.

Increased daytime frequency: complaint of voiding too often by day (usually ten or more voids when awake). Increased daytime frequency may arise in the presence of a normal bladder capacity when there is excessive fluid intake, or when bladder capacity is restricted secondary to detrusor overactivity, impaired bladder compliance, or increased bladder sensation ${ }^{3}$.

Nocturia: complaint that the individual has to wake at night more than one time to void and usually the patient wakes from sleep to pass urine. Nocturia may arise for similar reasons to daytime frequency, but may also occur due to an increase in fluid output due to other physiological abnormalities resulting in 'nocturnal polyuria's.

Nocturnal enuresis: complaint of loss of urine occurring during sleep ${ }^{3}$. Enquiry should include previous childhood nocturnal enuresis as delayed bladder control in childhood is associated with detrusor overactivity in adulthood.

\section{Initial assessment}

History of the lower urinary tract symptoms should be further described by specifying relevant factors such as type, frequency, severity, precipitating factors, social impact, effect on hygiene and quality of life, the measures used to contain the leakage (wearing of protection, number and type of pads and change of underwear or outer clothes) and whether or not the individual seeks or desires help because of urinary incontinence.

As there is subjective variation in assessing symptoms, ICI recommends the use of validated patient reported outcome questionnaires. Selfcompleted validated questionnaires also enable the clinician to compare the efficacy of the treatment and also useful for research purposes.

There are many different validated questionnaires to assess the lower urinary tract symptoms. Kings Health Questionnaire (KHQ) ${ }^{4}$ is one of the widely used questionnaires and is available in 39 languages (Annexure, page 132-4). It has demonstrated reliability and validity in assessing LUTS in both men and women. KHQ consists of three parts. The first section contains two questions measuring general health and overall health related to urinary symptoms. The second section includes 19 questions divided in to seven domains of quality of life: incontinence impact, role limitations, physical limitations, social limitations, personal relationships, emotions, sleep and energy and severity coping measures. The third section comprises of 11 questions measuring the bother or impact of urinary symptoms.

Other symptoms related to pelvic floor dysfunction also must be included in the history. These are the symptoms of pelvic organ prolapse (vaginal lump, heaviness, voiding dysfunction), bowel symptoms (faecal incontinence, faecal soiling, constipation, incomplete bowel emptying and digitation) and sexual dysfunction (superficial or deep dyspareunia, coital incontinence).

Patient should be questioned about any alteration in sensation and motor power in the legs or perineum, lack of bladder sensation and lower backache. Worsening symptoms of back pain with urinary and neurological symptoms may indicate a worsening central intervertebral disc prolapse. LUTS can also be the result of cerebrovascular accidents, Parkinson's disease, multiple sclerosis or peripheral neuropathy associated with diabetes mellitus.

It is important to enquire about the LUTS during the menstrual cycle. Presence of uterine fibroids can give rise to urinary frequency and voiding dysfunction. Previous gynaecological surgery may cause damage to urethral sphincter innervation and distortion or narrowing of the bladder neck. These may influence the success of future continence procedures.

The obstetric history should include parity, length of labour, mode of delivery, perineal trauma and the weight of the largest infant. Caesarean section or epidural analgesia during labour and subsequent postpartum urinary retention are possible predictors of subsequent voiding dysfunction.

Many drugs affect the lower urinary tract. Diuertics can produce urgency, frequency and urge urinary incontinence. Sedatives such as benzodiazepines may cause confusion and secondary incontinence particularly in elderly patients. Anticholinergics effect of some antipsychotic drugs, antidepressants, opiates, antiparkinsonian drugs and antispasmodics impair detrusor contractility and may cause voiding dysfunction, urinary retention with secondary overflow incontinence. 


\section{Examination}

Before examining the patient it is important to reassure her about the possibility of urinary leakage and explain that she should not be embarrassed as a result of this. The patient's mobility and mental state is important in her ability to react to the incontinence and may influence the management especially in elderly. Body mass index (BMI) must be noted at the first consultation as increased BMI is associated with LUTS and may also hinder the success of subsequent treatment.

Abdominal inspection will reveal evidence of previous abdominal surgery and palpation may demonstrate the presence of full or distended bladder as well as other pelvic masses.

It is important to perform a basic neurological examination testing the tone, strength and movement of the lower limbs and perineal and perianal sensation.

Inspection of vulval skin is important as there may be signs of excoriation, oedema or erythema due to prolonged exposure skin to urine and associated candidiasis. If the bladder is full stress urinary incontinence may be demonstrable. Pelvic organ prolapse is best assessed with an empty bladder in left lateral position with the use of a Sim's speculum. Examination in standing position is necessary if there is no obvious prolapse on left lateral position but the patient complains of symptoms.

Examination of urethra may reveal urethral mucosal prolapse, urethral caruncle. Urethral diverticulum may present with a midline anterior vaginal wall swelling or tenderness over the length of urethra. Vaginal cysts such as Gartner's duct cyst may present with similar findings.
A bimanual examination should be performed to assess the uterine size and to exclude abnormal pelvic masses or uterine impaction. Rectal examination is particularly important in elderly to exclude faecal impaction, which can aggravate urinary incontinence. Pelvic floor muscle tone can be assessed by observation and digital palpation circumferentially at rest and voluntary contraction.

\section{Conclusion}

Initial assessment of a patient with urinary incontinence must include a validated patient reported symptom questionnaire, detailed focused history and a systematic clinical examination. This can guide the direction of further investigations and management.

Second part of this review will be investigations of female urinary incontinence.

\section{References}

1. Abrams P, Cardozo L, Khoury S, Wein A. eds. Incontinence - 4th International Consultation on Incontinence. 4th Ed. Paris: Health Publication Ltd, 2009; 259.

2. Vasan SS, Yalaburgi R. Epidemiology urinary incontinence in Asia. In: Cardozo L, Staskin D (eds). Text Book of Female Urology and Urogynaecology. Essex: Informa Healthcare 2010; 6: 44-45.

3. Abrams P, Cardozo L, Fall M, Griffith D, et al. The standardisation of terminology of lower urinary function Report from the Standardisation Sub-committee of the International Continence Society. Neururology and Urodyna

4. Kelleher CJ, Cardozo LD, Khullar V, Salvatore S. A new questionnaire to assess the quality of life of urinary incontinent women. Br J Obstet Gynaecol 1997; 104: 1374-9. 


\section{The King's Health Questionnaire}

Annexure

1. How would you describe your health at the present? Please tick one answer

Very good

Good

Fair

Poor

Very poor

2. How much do you think your bladder problem affects your life? Please tick one answer

Not at all

A little

Moderately

A lot

Below are some daily activities that can be affected by bladder problems.

How much does your bladder problem affect you?

We would like you to answer every question. Simply tick the box that applies to you

\section{ROLE LIMITATIONS}

Not at all

Slightly

Moderately

A lot

A. Does your bladder problem affect your household tasks? (cleaning, shopping etc)

B. Does your bladder problem affect your job, or your normal daily activities outside the home?

\section{PHYSICAL / SOCIAL LIMITATION}

Not at all

Slightly

Moderately

A lot

A. Does your bladder problem affect your physical activities (e.g. going for a walk, running, sport, gym etc)?

B. Does your bladder problem affect your ability to travel?

C. Does your bladder problem limit your social life?

D. Does your bladder problem limit your ability to see and visit friends?

\section{PERSONAL RELATIONSHIPS}
Not at all
Slightly
Moderately
A lot
A. Does your bladder problem affect your relationship with your partner?
B. Does your bladder problem affect your sex life?
C. Does your bladder problem affect your family life?

Applicable

\section{EMOTIONS}

Not at all

Slightly

Moderately

Very much

A. Does your bladder problem make you feel depressed?

B. Does your bladder problem make you feel anxious or nervous?

C. Does your bladder problem make you feel bad about yourself? 
7. SLEEP/ENERGY

Never $\square$ Sometimes $\square$ Often $\square$ All the time $\square$

A. Does your bladder problem affect your sleep?

B. Does your bladder problem make you feel worn out and tired ?

8. Do you do any of the following? If so how much?

Never $\square$ Sometimes $\square$ Often $\square$ All the time $\square$
A. Wear pads to keep dry?
B. Be careful how much fluid you drink?
C. Change your underclothes because they get wet?
D. Worry in case you smell?

We would like to know what your bladder problems are and how much they affect you? From the list below choose only those problems that you have at present.

Leave out those that don't apply to you.

How much do they affect you?

FREQUENCY: going to the toilet very often
1. A little
2. Moderately
3. A lot

NOCTURIA: getting up at night to pass urine
1. A little
2. Moderately
3. A lot

URGENCY: a strong and difficult to control desire to pass urine
1. A little
2. Moderately
3. A lot

URGE INCONTINENCE: urinary leakage associated with a strong desire to pass urine
1. A little
2. Moderately
3. A lot

STRESS INCONTINENCE: urinary leakage with physical activity eg. coughing, running
1. A little
2. Moderately
3. A lot

NOCTURNAL ENURESIS: wetting the bed at night
1. A little
2. Moderately
3. A lot

INTERCOURSE INCONTINENCE: urinary leakage with sexual intercourse
1. A little
2. Moderately
3. A lot

WATERWORKS INFECTIONS
1. A little
2.Moderately
3. A lot

BLADDER PAIN
1. A little
2.Moderately
3. A lot

Thank You For Your Time 


\section{To Calculate Scores}

\section{PART 1}

1) General Health Perceptions

Very good 1

Good 2

Fair 3

Poor 4

Very poor 5

Score $=(($ Score to $\mathrm{Q} 1-1) / 4) \times 100$

2) Incontinence Impact

Not at all 1

A little 2

Moderately 3

A lot 4

Score $=(($ Score to $Q 2-1) / 3) \times 100$

\section{PART 2}

Individual scores as recorded at the top of each column of possible responses

3) Role limitations

Score $=((($ Scores to $Q 3 A+3 B)-2) / 6) \times 100$

4) Physical limitations

Score $=((($ Scores to $Q 4 A+4 B)-2) / 6) \times 100$

5) Social limitations

[If $5 \mathrm{C}>/=1]$ Score $=((($ Score to $\mathrm{Q} 4 \mathrm{C}+4 \mathrm{D}+5 \mathrm{C})-3) / 9) \times 100$

[If $5 \mathrm{C}=0$ ] Score $=((($ Score to $\mathrm{Q} 4 \mathrm{C}+4 \mathrm{D})-2) / 6) \times 100$

6) Personal relationships

[If $5 A+5 B>=2]$ Score $=((($ Scores to $Q 5 A+5 B)-2) / 6) \times 100$

[If $5 \mathrm{~A}+5 \mathrm{~B}=1$ ] Score $=((($ Scores to $\mathrm{Q} 5 \mathrm{~A}+5 \mathrm{~B})-1) / 3) \times 100$

[If $5 \mathrm{~A}+5 \mathrm{~B}=0$ ] Treat as missing value

7) Emotions

Score $=((($ Score to $Q 6 A+6 B+6 C)-3) / 9) \times 100$

8) Sleep / energy

Score $=((($ Scores to $Q 7 A+7 B)-2) / 6) \times 100$

9) Severity measures

Score $=((($ Scores to $Q 8 A+8 B+8 C+8 D)-4) / 12) \times 100$

\section{PART 3}

Scale score

Omitted 0

A little 1

Moderately 2

A lot 3 